Bull. Austral. Math. Soc.

VOL. $62(2000)$ [307-310]

\title{
FINDING COMMON FIXED POINTS OF NONEXPANSIVE MAPPINGS BY ITERATION
}

\author{
B.E. RHOADES
}

In this paper it is shown that a particular iteration scheme converges weakly to a common fixed point of a finite set of nonexpansive mappings. This result is an improvement of two related theorems in the literature.

Let $X$ be a Banach space, $C$ a convex subset of $X$. Let $T_{1}, T_{2}, \ldots, T_{k}$ be a family of nonexpansive selfmaps of $C$. Kuhfittig [4] defined the following iteration scheme. Let $U_{0}=I, I$ the identity map, $0<\alpha<1$,

$$
\begin{aligned}
& U_{1}=(1-\alpha) I+\alpha T_{1} U_{0}, \\
& U_{2}=(1-\alpha) I+\alpha T_{2} U_{1},
\end{aligned}
$$

$$
\begin{aligned}
& U_{k}=(1-\alpha) I+\alpha T_{k} U_{k-1}, \\
& x_{0} \in C, x_{n+1}=(1-\alpha) x_{n}+\alpha T_{k} U_{k-1} x_{n}, n \geqslant 0 .
\end{aligned}
$$

Define $F=\bigcap_{i=1}^{k} F\left(T_{i}\right)$, where $F\left(T_{i}\right)$ denotes the fixed point set of $T_{i}$. Then, if each $T_{i}$ is a nonexpansive selfmap of $C$, with $F \neq \emptyset, C$ compact, $X$ strictly convex, Kuhfittig [4] showed that (1) converges strongly to a common fixed point of the family. His second result is that, if $X$ is uniformly convex and satisfies Opial's condition and $C$ is closed and convex, then (1) converges weakly to a fixed point in $F$.

A Banach space is said to satisfy Opial's condition if, whenever $\left\{x_{n}\right\}$ is a convergent sequence in $X$ with limit $x_{0}$, then, for any $x \neq x_{0}$

$$
\liminf _{n \rightarrow \infty}\left\|x_{n}-x_{0}\right\|<\liminf _{n \rightarrow \infty}\left\|x_{n}-x\right\| .
$$

The purpose of this note is to improve [4, Theorem 2] by removing the hypothesis of Opial's condition.

The result to be proved is the following.

Received 17th February, 2000

Copyright Clearance Centre, Inc. Serial-fee code: 0004-9727/00 \$A2.00+0.00. 
THEOREM . Let $X$ be a uniformly convex Banach space, $C$ a closed convex subset of $X, T_{1}, T_{2}, \ldots, T_{k}$ a family of nonexpansive selfmaps of $C$ with $F \neq \emptyset$. Then $\left\{x_{n}\right\}$, defined by (1), converges weakly to a common fixed point of the family.

The proof of the Theorem will require the following lemmas.

Let $C$ be a subset of a Banach space $X, T: C \rightarrow X, x_{0} \in C$. Then the Mann iterative scheme $M\left(x_{0}, t_{n}, T\right)$ is the sequence $\left\{x_{n}\right\}$ defined by $x_{n+1}=\left(1-t_{n}\right) x_{n}+$ $t_{n} T x_{n}$. If the sequence $\left\{t_{n}\right\}$ satisfies $0 \leqslant t_{n} \leqslant b<1, \sum t_{n}=\infty$, and $x_{n} \in C$ for each positive integer $n$, then $M\left(x_{0}, t_{n}, T\right)$ is said to satisfy condition $A$.

LEMma 1. [3, Lemma 2] Let $C$ be a subset of a Banach space $X, T$ a nonexpansive map from $C$ into $X$. If $M\left(x_{0}, t_{n}, T\right)$ satisfies condition $A$ and is bounded, then $\lim \left\|x_{n}-T x_{n}\right\|=0$.

Let $C$ be a closed bounded convex subset of a uniformly convex space $X$. A map $T$ is said to be semicontractive if there exists a map $V: C \times C \rightarrow X$ such that $T(u)=V(u, u)$ for each $u \in C$ while, (a) for each fixed $v$ in $C, V(\cdot, v)$ is nonexpansive from $C$ to $X$, and (b) for each fixed $u \in C, V(u, \cdot)$ is completely continuous from $C$ to $X$ uniformly for $u$ in bounded subsets of $C$.

LEMma 2. [2, Theorem 3] Let $X$ be uniformly convex, $C$ a closed bounded convex subset of $X, T$ a semicontractive mapping of $C$ into $X$. Then:

(a) $(I-T)$ is demiclosed, and

(b) $(I-T)(C)$ is closed in $X$.

ProOF of THE TheOREM: From [4], the $U_{i}$ and $T_{i} U_{i-1}$ are nonexpansive and $\left\{T_{1}, T_{2}, \ldots, T_{k}\right\}$ and $\left\{U_{1}, U_{2}, \ldots, U_{k}\right\}$ have the same fixed point set. Let $p \in F$, set $S=T_{k} U_{k-1}$.

For any $x \in C, p \in F$, define $E=\{u \in X:\|u-p\| \leqslant r\} \cap C$, where $r:=\|x-p\|$. Then $E$ is a nonempty bounded convex subset of $C$ which is invariant under the $U_{i}$ and $T_{i}$ and contains $x_{0}=x$. Thus, without loss of generality we may assume that $C$ is bounded.

Since $S$ is nonexpansive, using (1),

$$
\begin{aligned}
\left\|x_{n+1}-p\right\| & =\left\|(1-\alpha) x_{n}+\alpha S x_{n}\right\| \leqslant(1-\alpha)\left\|x_{n}-p\right\|+\alpha\left\|S x_{n}-p\right\| \\
& \leqslant(1-\alpha)\left\|x_{n}-p\right\|+\alpha\left\|x_{n}-p\right\|=\left\|x_{n}-p\right\| .
\end{aligned}
$$

Therefore $\lim \left\|x_{n}-p\right\|$ exists, which implies that $\left\{x_{n}\right\}$ is bounded.

From Lemma 1, $\lim _{n}\left\|x_{n}-S x_{n}\right\|=0$.

The assumption that $X$ is uniformly convex implies that it is reflexive. The boundedness of $\left\{x_{n}\right\}$ implies that there is a subsequence $\left\{x_{n_{i}}\right\}$ of $\left\{x_{n}\right\}$ which converges weakly to a point $q \in C$. Since $S$ is nonexpansive, if one defines $V$ by $V(u, v)=S u+v$, 
then $V$ is semicontractive, and, from Lemma 2, $S$ is demiclosed. This means that, if $\left\{x_{n_{i}}\right\}$ converges weakly to a point $q$, then, since $\lim _{i}\left\|(I-S) x_{n_{i}}\right\|=0,(I-S) q=q$; that is, $q$ is a fixed point of $S=T_{k} U_{k-1}$.

A uniformly convex space is strictly convex, so one can use the argument of [4], which we now do, to show that $q \in F$.

Suppose that $q$ is not a common fixed point of $T_{k-1}$ and $U_{k-2}$. Then the closed line segment $\left[q, T_{k-1} U_{k-2} q\right]$ has positive length. Define

$$
z=U_{k-1} q=(1-\alpha) q+\alpha T_{k-1} U_{k-2} q
$$

By hypothesis there exists a point $w$ such that $T_{1} w=T_{2} w=\cdots=T_{k} w=w$. Since $\left\{T_{i}\right\}$ and $\left\{U_{i}\right\}$ have the same fixed point set, it follows that $T_{k-1} U_{k-2} w=w$. Since $T_{k-1}$ and $U_{k-2}$ are nonexpansive,

$$
\left\|T_{k-1} U_{k-2} q-w\right\| \leqslant\|q-w\|
$$

and

$$
\left\|T_{k} z-w\right\| \leqslant\|z-w\| .
$$

Therefore $w$ is at least as close to $T_{k} z$ as to $z$. But $T_{k} z=T_{k} U_{k-1} q=q$, so that $w$ is at least as close to $q$ as to $z=(1-\alpha) q+\alpha T_{k-1} U_{k-2} q$. Since $X$ is strictly convex, it follows that

$$
\|q-w\|<\left\|T_{k-1} U_{k-2} q-w\right\|,
$$

contradicting (2), so that $T_{k-1} U_{k-2} q=q$. It then follows from $U_{k-1}=(1-\alpha) I+$ $\alpha T_{k-1} U_{k-2}$ that $U_{k-1} q=(1-\alpha) q+\alpha q=q$ and $q=T_{k} U_{k-1} q=T_{k} q$. Therefore $q$ is a common fixed point of $T_{k}$ and $U_{k-1}$.

Since $T_{k-1} U_{k-2} q=q$, we may repeat the above argument to obtain the result that $T_{k-2} U_{k-2} q=q$ and that $q$ is therefore a common fixed point of $T_{k-1}$ and $U_{k-2}$. Continuing in the same manner, it then follows that $T_{1} U_{0} q=q$ and $q$ is a common fixed point of $T_{2}$ and $T_{1}$. Thus $q$ is a common fixed point of $\left\{T_{i}: i=1,2, \ldots, k\right\}$.

COROLlary . [4, Theorem 2] If $X$ is a uniformly convex Banach space satisfying Opial's condition and $C$ is a closed convex subset of $X$, and if the family of mappings $\left\{T_{i}: i=1,2, \ldots, k\right\}$ satisfies (1), then, for any $x \in C$, the sequence $\left\{x_{n}\right\}$ converges weakly to a common fixed point.

In a recent paper, Atsushiba and Takahashi [1] proved the following.

THEOREM AT . Let $X$ be a uniformly convex Banach space which satisfies Opial's condition or whose norm if Fréchet differentiable. Let $C$ be a nonempty closed convex 
subset of $X, S$ and $T$ a pair of commuting nonexpansive selfmaps of $C$, with $F(S) \cap$ $F(T) \neq \emptyset$. Let $x_{1} \in C$ and define $\left\{x_{n}\right\}$ by

$$
x_{n+1}=\alpha_{n} x_{n}+\left(1-\alpha_{n}\right) \frac{1}{n^{2}} \sum_{i, j=0}^{n} S^{i} T^{j} x_{n} \text { for } n \in \mathbb{N}
$$

where $\left\{\alpha_{n}\right\}$ satisfies $0 \leqslant \alpha_{n} \leqslant a<1$. Then $\left\{x_{n}\right\}$ converges weakly to a common fixed point of $T$ and $S$.

Iteration scheme (1) is much simpler than (3). In addition, the theorem of this paper shows that (1) is a much more general iteration scheme than (3), since the hypotheses of Opial's condition and commutativity of the maps are not required.

Finally we note that, in (1) one can replace $\alpha$, in the formula for $x_{n+1}$, with a sequence $\left\{t_{n}\right\}$, satisfying Condition $\mathrm{A}$.

\section{REFERENCES}

[1] S. Atsushiba and W. Takahashi, 'Approximating common fixed points of two nonexpansive mappings in Banach spaces', Bull. Austral. Math. Soc. 57 (1999), 117-127.

[2] F.E. Browder, 'Some contractive and semiaccretive nonlinear mappings in Banach spaces', Bull. Amer. Math. Soc. 74 (1968), 660-665.

[3] S. Ishikawa, 'Fixed points and iteration of a nonexpansive mapping in a Banach space', Proc. Amer. Math. Soc. 59 (1976), 65-71.

[4] P.K.F. Kuhfittig, 'Common fixed points of nonexpansive mappings by iteration', Pacific J. Math. 97 (1981), 137-139. 